\title{
Erratum to: The relationship between sodium concentrations in spot urine and blood pressure increases: a prospective study of Japanese general population: the Circulatory Risk in Communities Study (CIRCS)
}

Mitsumasa Umesawa ${ }^{1,2}$, Kazumasa Yamagishi ${ }^{2}$, Hiroyuki Noda ${ }^{3}$, Ai Ikeda $^{4}$, Shinobu Sawachi ${ }^{2,5}$, Isao Muraki ${ }^{6}$, Choy-Lye Chei ${ }^{2}$, Renzhe Cui ${ }^{3}$, Masanori Nagao ${ }^{1}$, Tetsuya Ohira ${ }^{3}$, Tomoko Sankai ${ }^{7}$, Takeshi Tanigawa ${ }^{4}$, Akihiko Kitamura $^{3}$, Masahiko Kiyama ${ }^{6}$, Hiroyasu Iso $^{3^{*}}$ and CIRCS Investigators

\section{Erratum}

Unfortunately, the original version of this article [1] contained an error.

We would like to correct a formula in "Validation study" section in page 3 .

We wrote that "sodium excretion $(\mathrm{g} /$ day $)=0.032 \mathrm{x}$ sodium concentration ( $\mathrm{mmol} / \mathrm{l}$ ) -2.278 (women) + 9.130", however, we would like to replace this with " $\mathrm{NaCl}$ excretion $(\mathrm{g} /$ day $)=0.032 \mathrm{x}$ sodium concentration $(\mathrm{mmol} / \mathrm{l})-2.278$ (women) + 9.130".

\section{Author details}

'Department of Public Health, Dokkyo Medical University, School of Medicine, 880 Kita-kobayashi, Mibu, Shimotsuga-gun, Tochigi 321-0293, Japan. ${ }^{2}$ Department of Public Health Medicine, Faculty of Medicine, University of Tsukuba, 1-1-1 Tennodai, Tsukuba 305-8575, Japan. ${ }^{3}$ Public Health, Department of Social Medicine, Osaka University Graduate School of Medicine, 2-2 Yamadaoka, Suita 565-0871, Japan. ${ }^{4}$ Department of Public Health, Graduate School of Medicine, Juntendo University, 2-1-1 Hongo, Bunkyo-ku, Tokyo 113-8421, Japan. ${ }^{5}$ Biogen Japan Ltd., 14th floor 1-4-1 Nihonbashi, Chuo-ku, Tokyo 103-0027, Japan. ${ }^{6}$ Osaka Center for Cancer and Cardiovascular Diseases Prevention, 1-3-2 Nakamichi, Higashinari-ku, Osaka 537-0025, Japan. ${ }^{7}$ Department of Community Health, Faculty of Medicine, University of Tsukuba, 1-1-1 Tennodai, Tsukuba 305-8575, Japan.
Received: 8 June 2016 Accepted: 9 June 2016

Published online: 15 June 2016

\section{Reference}

1. Umesawa $\mathrm{M}$ et al. The relationship between sodium concentrations in spot urine and blood pressure increases: a prospective study of Japanese general population: the Circulatory Risk in Communities Study (CIRCS). BMC Cardiovasc Disord. 2016;16:55.

\footnotetext{
* Correspondence: iso@pbhel.med.osaka-u.ac.jp

${ }^{3}$ Public Health, Department of Social Medicine, Osaka University Graduate

School of Medicine, 2-2 Yamadaoka, Suita 565-0871, Japan

Full list of author information is available at the end of the article
} 